\title{
Interior Design Scheme Recommendation Method Based on Improved Collaborative Filtering Algorithm
}

\author{
Wei He \\ School of Design and Art, Xijing University, Xi'an, Shaanxi 710123, China \\ Correspondence should be addressed to Wei He; 20070044@xijing.edu.cn
}

Received 20 October 2021; Revised 8 November 2021; Accepted 17 November 2021; Published 23 December 2021

Academic Editor: Junjuan Xia

Copyright (C) 2021 Wei He. This is an open access article distributed under the Creative Commons Attribution License, which permits unrestricted use, distribution, and reproduction in any medium, provided the original work is properly cited.

\begin{abstract}
The recommendation engine is similar to the function of the product recommender in our real life, which provides great convenience for people to choose the appropriate decoration scheme in the process of interior design and decoration. A home improvement website or company can design a suitable recommendation algorithm to provide home improvement program recommendation services for users with decoration needs. After understanding the user behavior of the home decoration website, this paper proposes an interior design scheme recommendation method based on an improved collaborative filtering algorithm. The method designs a collaborative filtering algorithm that combines multilayer hybrid similarity and trust mechanisms. Fuzzy set membership function is introduced to correct users' rating similarity, and users' interest vector is extracted to calculate users' preference for different types of items. The algorithm dynamically fuses those two aspects to obtain the mixed similarity of users; meanwhile, the user's hybrid similarity and trust are fused in an adaptive model. Then, the user neighbor data set generated based on the overall similarity of users is used as a training set, taking the item scores and features into consideration. On the one hand, the users and the projects are taken into account as well. The final prediction score is more accurate, and the recommendation effect is better. The experimental results show that this method can recommend interior design schemes with high performance, and its performance is better than other methods.
\end{abstract}

\section{Introduction}

With the sustained and rapid growth of China's national economy and the increasing per capita income, coupled with the strong promotion of China's real estate industry, the demand of our interior design and decoration industry has remained strong. The rapid development of the Internet has also promoted people's participation and demand on the Internet. The number of Internet users has increased to a considerable degree. Online shopping has penetrated into every aspect of our daily life. Amazon, Taobao, Jingdong and many other websites are relatively successful and mature e-commerce platforms, while home decoration e-commerce has not yet become mature. As the online shopping experience of home decoration is not high, users' purchasing desire is not strong. While the consumption of home decoration is increasingly hot in recent years, it is relatively backward in ecommerce [1].
The domestic decoration market economy scale has exceeded 4 trillion yuan. The number of home decoration consumers is huge, but the proportion of online shopping in the total consumption of the home decoration market is relatively small. Home decoration websites record behavior logs of hundreds of millions of users [2]. However, the main task of this paper is how to automatically locate and push the home decoration scheme that users are interested in from the massive information.

A home outfit plan covers the design idea of the stylist. When it is accepted and adopted by decoration users, the plan will involve the interests of users themselves, furniture companies, decoration companies, designers, home decoration websites, and other aspects [3]. The server can record user behavior logs when users browse, click, or download home decoration solutions. If their favorite home decoration scheme from the growing user behavior log can be dug out and recommended to users, the decoration needs of users 
will be met and a good reputation be established, with more value being brought to home decoration websites and related enterprise chains.

The online behavior of the home outfit industry is moving towards scale gradually. Literatures $[4,5]$ have begun to design home decoration products based on user perception. Literature [6] proposes a new method to extract and retrieve furniture items from image databases and online websites containing multiple furniture items. This method supports finding similar items from the database. Consumers usually pay the most attention to the pictures, prices, and brand reputation evaluation of home improvement programs when shopping online for home improvement [7]. Poor user experience and the fact of not being able to find a satisfactory home improvement plan within a certain period of time are major weaknesses of domestic home improvement online consumption, which bring huge economic loss to home outfit network shopping. The recommendation system is a good solution. However, the problems of massive log processing efficiency and recommendation accuracy brought by the rapidly increasing number of users are important factors restricting the application of the recommendation system.

The concept of the recommendation system, which was gradually applied to the commercial field after a period of time, was first formally proposed by Varian and Resnick. Nowadays, recommendation has developed into a very popular research field [8]. Collaborative filtering algorithm is widely used in the research of recommendation system algorithm [9]. However, it is found that the traditional collaborative filtering algorithm still has many problems to be improved with the deepening of research [10]. Researchers have studied the improved algorithm. The key to the improved algorithm in Ref. [11] was the calculation of semantic similarity and the acquisition of similarity word frequency weight. Reference [12] proposed an improved metric. The method considers the ratio between the absolute score value and the number of common scoring items. Literature [13] proposed that the recommendation results could be more reliable by mining the potential relationship between rating users. By comprehensively analyzing the user's historical behavior and item label information, literature [14] realized the recommendation of label probability without relying on the user's item score. Literature [15] used genetic algorithm to find the best item list for users. Most of the objects studied by these recommendation algorithms are for music, movies and digital products, etc., but there are few recommendation system algorithms specifically for the consumption behavior of home decoration websites. And it is even rarer to apply the recommendation algorithm to practical applications. Literature [16] mentioned interior design recommendation technology based on collaborative filtering algorithm. The literature only gives a framework, but does not give the actual recommendation algorithm and application.

Aiming at the above problems, this paper applies the improved collaborative filtering algorithm to the design of interior design recommendation technology. This method provides accurate interior design recommendation service for users by integrating user behavior and project behavior. Interior design recommendation technology has low operational requirements for users and plays an important role in enhancing user experience. The results show that the proposed method solves the limitations of the current interior design project recommendation method, and it is a project recommendation method with high precision and high speed.

\section{Related Research}

The collaborative filtering (CF) algorithm uses past behavior or the opinions of existing user groups to predict data and also uses neighborhood data similar to the current user or project to generate recommendations. The basic steps include data collection and processing, generation of a user-item score matrix, calculation of similarity, generation of the nearest neighbor, prediction of score, and generation of a top $N$ recommendation.

2.1. User-Based Collaborative Filtering Algorithm (UserCF). Firstly, the score data set and the current user ID are entered to find other users with similar preferences to the current user. These users are called nearest neighbors. Then, the ratings of all items are ranked from highest to lowest using the ratings of the neighbor user-predicted items. Finally, the top $N$ items are recommended to the current user. The algorithm flow in this paper is shown in Figure 1.

Similarity measurement is the core of the collaborative filtering algorithm, including cosine similarity, modified cosine similarity, and Pearson similarity. Pearson correlation coefficient is used to calculate the linear correlation between two variables, and the formula is as follows:

$$
\operatorname{correlation}(m, n)=\frac{\sum_{k \in I_{m n}}\left(s_{m k}-\bar{s}_{m}\right) \times\left(s_{n k}-\bar{s}_{n}\right)}{\sqrt{\sum_{k \in I_{m n}}\left(s_{m k}-\bar{s}_{m}\right)^{2}} \times \sqrt{\sum_{k \in I_{m n}}\left(s_{n k}-\bar{s}_{n}\right)^{2}}},
$$

where correlation $(m, n)$ represents the similarity between users $m$ and $n$. $I_{m n}$ represents the set of goods jointly rated by users $m$ and $n$, while $s_{m k}$ and $s_{n k}$ represent the scores on goods $k . \bar{s}_{m}$ and $\bar{s}_{n}$ represent the average rating of the product by users $m$ and $n$, respectively.

The scoring prediction formula is defined as follows:

$$
P_{m k}=\bar{s}_{m}+\frac{\sum_{n \in I_{m}} \operatorname{sim}(m, n) \times\left(s_{n k}-\bar{s}_{n}\right)}{\sum_{n \in I_{m}} \operatorname{sim}(m, n)},
$$

where $\bar{s}_{m}$ represents the average score of all rated products by target user $m$. $I_{m}$ indicates the neighbor set of the target user. $s_{n k}$ represents the neighbor user's rating of item $k$. $\bar{s}_{n}$ represents the average value of ratings of the items rated by the neighbor users. $\operatorname{sim}(m, n)$ indicates the similarity between the target user and the neighbor user.

Although the UserCF algorithm has been widely used in the field of recommendation, it also faces many challenges. For e-commerce websites, the number of projects is relatively stable, but the number of users is updated at a high 


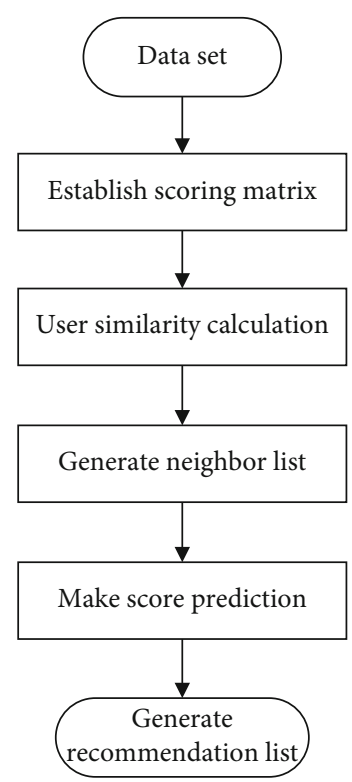

Figure 1: User-based collaborative filtering algorithm flow.

frequency. When the number of users is much larger than the number of items, computing similarities between users becomes increasingly time-consuming and takes up more memory. The recommendation results generated by UserCF algorithms are poorly interpretable.

\subsection{Item-Based Collaborative Filtering Algorithm (ItemCF).} Firstly, an item similarity matrix is constructed to describe the similarity between two items. $k$ nearest neighbor projects similar to the current project are found. The user score for each item $m$ not seen by the current user is then calculated based on $k$ nearest neighbors. Finally, the user's ratings for all items are sorted from large to small, and the top $N$ items with the highest scores are recommended to the current user.

The basic steps of the item-based collaborative filtering algorithm (ItemCF) are processing data, generating useritem score matrix, computing item similarity, generating nearest neighbor, predicting score, and generating top $N$ recommendation.

Then, cosine similarity is used to calculate the similarity between the two items. In addition, based on the $k$ nearest neighbor projects acquired, the current user's interest in the target project is predicted.

\section{Interior Design Scheme Recommendation Based on Improved Collaborative Filtering Algorithm}

The traditional collaborative filtering recommendation algorithm only generates recommendations based on the user's historical score data. CF only considers scoring data, which not only is incomplete but also leads to cold start problems. This makes new projects or new users unable to recommend according to the collaborative filtering recommendation algorithm. At the same time, it will also bring serious data sparsity problems and affect the accuracy of recommendation results. Therefore, this paper presents an interior design scheme recommendation method based on an improved collaborative filtering algorithm. This method considers multidimensional mixed similarity, trust mechanism, and item scoring, which make the final prediction score more accurate and the recommendation effect better.

3.1. User Feature Extraction. For users or interior design projects, they all have their own characteristics. In the design process of the indoor project design project recommendation method based on the improved collaborative filtering algorithm, the user characteristics have a certain stability. For two users with similar characteristics, the probability of selecting similar interior design projects is higher. Firstly, the characteristics of interior design users are described, and then, they are quantified.

For the gender characteristics of interior design users, " 1 " represents male and " 1 " represents female, while the gender and age characteristics of interior design users are quantified by " $0-4$," where " 0 " represents under 30 years old, “1” represents 30-39, “2” represents 39-44, “ 3 ” represents 45-60, and " 4 " represents over 60 years old. The occupation and education level are also quantified by numbers, which will not be described in detail here.

\subsection{Design of Multidimensional Similarity and Trust Measurement}

3.2.1. Revise Score Similarity. In the recommendation system, the score of an item reflects the user's preference for the item to a certain extent. At this point, the measurement of preference degree is determined by the user rating value. But the ratings are not an accurate representation of how much users like them. For example, a movie is scored on a 5 -point scale. User $i$ gives a score of 4 . At this time, the user's preference for the movie cannot be accurately quantified in numerical form. It can only roughly predict the probability that user $i$ likes the movie with high probability. To solve the problem of measuring the degree of preference in this situation, this paper adopts the fuzzy logic method to enrich the single numerical score, so as to obtain the numerical quantification of user preference. The membership function definition of scoring triangle fuzzy set is shown in Figure 2.

$$
\begin{array}{ll}
\varphi_{\text {good }}(z)=, & 1 \leq z \leq 5, \\
\varphi_{\text {bad }}(z)=, & 1 \leq z \leq 5 .
\end{array}
$$

The calculation of preference difference between any two users depends on the rating difference between them for the common rated items. The greater the difference in ratings, the greater the degree of preference difference between them. The preference difference can be calculated by formula (3). Based on this inference, the preference similarity of any two users calculated by using public 


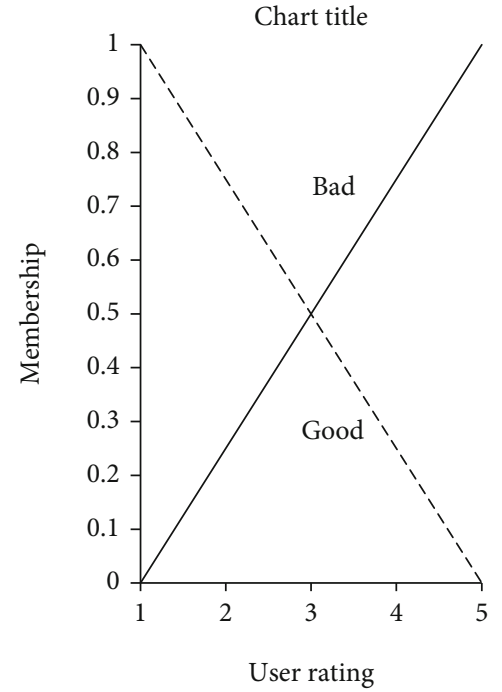

FIgURE 2: Membership function of scoring triangular fuzzy.

TABLE 1: MAE values under different interest vector weights.

\begin{tabular}{lcccccc}
\hline$\varepsilon$ & 10 & 20 & 30 & 40 & 50 & 60 \\
\hline 0 & 0.7550 & 0.7418 & 0.7338 & 0.7310 & 0.7249 & 0.7233 \\
0.1 & 0.7540 & 0.7420 & 0.7351 & 0.7317 & 0.7256 & 0.7237 \\
0.2 & 0.7549 & 0.7397 & 0.7346 & 0.7313 & 0.7243 & 0.7228 \\
0.3 & 0.7559 & 0.7389 & 0.7349 & 0.7330 & 0.7257 & 0.7237 \\
0.4 & 0.7549 & 0.7409 & 0.7349 & 0.7308 & 0.7260 & 0.7231 \\
0.5 & 0.7570 & 0.7418 & 0.7349 & 0.7317 & 0.7244 & 0.7226 \\
0.6 & 0.7539 & 0.7408 & 0.7358 & 0.7320 & 0.7239 & 0.7218 \\
0.7 & 0.7559 & 0.7398 & 0.7366 & 0.7297 & 0.7249 & 0.7208 \\
0.8 & 0.7569 & 0.7420 & 0.7367 & 0.7289 & 0.7259 & 0.7229 \\
0.9 & 0.7598 & 0.7428 & 0.7397 & 0.7318 & 0.7290 & 0.7249 \\
1 & 0.7605 & 0.7467 & 0.7427 & 0.7376 & 0.7347 & 0.7307 \\
\hline
\end{tabular}

items is represented by $\operatorname{Ps}(m, n)$.

$$
\begin{gathered}
\operatorname{dis}\left(r_{m \text { com }}-r_{n \text { com }}\right)=\sqrt{\sum_{k=1}^{K}\left(r_{m \text { com }}^{k}-r_{n c o m}^{k}\right)^{2}}, \\
\operatorname{diff}(m, n)= \\
\operatorname{Ps}(m, n)=\frac{1}{(1+\operatorname{diff}(m, n))},
\end{gathered}
$$

where $r_{m \text { com }}$ and $r_{n c o m}$ represent the fuzzy preferences of any two users $m$ and $n$. $k$ represents the $k$ th element of vector $r_{m c o m}^{k} . K$ represents the dimension of the vector. $\operatorname{dis}\left(r_{m \text { com }}-r_{n \text { com }}\right) \quad$ represents the Euclidean distance between vectors. $\operatorname{diff}(m, n)$ is used to represent the mean difference degree of the scoring preference.

In the calculation of the user rating similarity $\operatorname{sim}_{a}(m, n)$ , user preference modified by introducing fuzzy mapping is combined with the Jaccard similarity of modified rating. This balances the dimensions of the user to the item and
TABle 2: MAE values under different dynamic weights.

\begin{tabular}{lcccccc}
\hline$\omega$ & 10 & 20 & 30 & 40 & 50 & 60 \\
\hline 0 & 0.7797 & 0.7597 & 0.7500 & 0.7444 & 0.7409 & 0.7387 \\
0.1 & 0.7771 & 0.7580 & 0.7484 & 0.7432 & 0.7399 & 0.7371 \\
0.2 & 0.7731 & 0.7578 & 0.7479 & 0.7416 & 0.7390 & 0.7369 \\
0.3 & 0.7710 & 0.7548 & 0.7459 & 0.7416 & 0.7372 & 0.7348 \\
0.4 & 0.7720 & 0.7537 & 0.7436 & 0.7390 & 0.7356 & 0.7336 \\
0.5 & 0.7699 & 0.7528 & 0.7428 & 0.7386 & 0.7342 & 0.7325 \\
0.6 & 0.7659 & 0.7488 & 0.7417 & 0.7375 & 0.7327 & 0.7295 \\
0.7 & 0.7636 & 0.7459 & 0.7375 & 0.7351 & 0.7318 & 0.7281 \\
0.8 & 0.7566 & 0.7467 & 0.7363 & 0.7321 & 0.7295 & 0.7258 \\
0.9 & 0.7599 & 0.7469 & 0.7366 & 0.7316 & 0.7288 & 0.7248 \\
1 & 0.7608 & 0.7479 & 0.7399 & 0.7368 & 0.7343 & 0.7284 \\
\hline
\end{tabular}

the proportion of items in the common rating among users. Finally, the user's score similarity is calculated based on the user's modified cosine similarity.

$$
\operatorname{sim}_{a}(m, n)=\operatorname{sim}(m, n)^{\operatorname{Adcos}} \times \operatorname{Ps}(m, n) \times \operatorname{Jaccard},
$$

where Jaccard $=\left|I_{m} \cap I_{n}\right| /\left|I_{m} \cup I_{n}\right| . I_{m}$ and $I_{n}$ represent the set of rated items for users $m$ and $n$.

3.2.2. User Interest Similarity. The traditional collaborative filtering algorithm only considers the user's project score value and ignores the similarity of the user's interest to different project types. However, users' interest similarity affects the recommendation accuracy of projects to a great extent. In the case of sparse data, there are few common scoring items among users, or even zero. At this point, user interest similarity evaluated based on project type can compensate for the impact of data sparsity to a large extent.

The calculation of the user's interest degree is affected by the scoring value of different item types and the proportion of quantity. For example, it is known that the user is interested in comedy and romance movies by analyzing and calculating a user's movie viewing history. Then, when a movie of a related type appears, it can be inferred that the user has a greater possibility to like the movie. The user interest vector interest ${ }_{m, n}$ is defined as the user's preference degree for different types of items based on the above deduction. The composition of the interest vector includes the proportion of the scoring value and the proportion of the scoring quantity of each item type.

$$
\begin{gathered}
\operatorname{avg}_{m}(q)=\frac{\sum_{m \in I_{q}} R_{m, c}}{\left|I_{q}\right|}, \\
\text { interest }_{m, n}=\varepsilon \frac{\operatorname{avg}_{m}(q)}{\sum_{z \in Q} \operatorname{avg}_{m}(z)}+(1-\varepsilon) \frac{N_{q}}{N},
\end{gathered}
$$

where $\operatorname{avg}_{m}(q)$ represents the average score of a certain type of item. $R_{m, c}$ represents the score of a certain type of item. $I_{q}$ represents the number of scoring for this type of item. $Q$ represents the collection of all types of items. $z$ represents a certain type of items. $N_{q}$ represents the number of scoring 


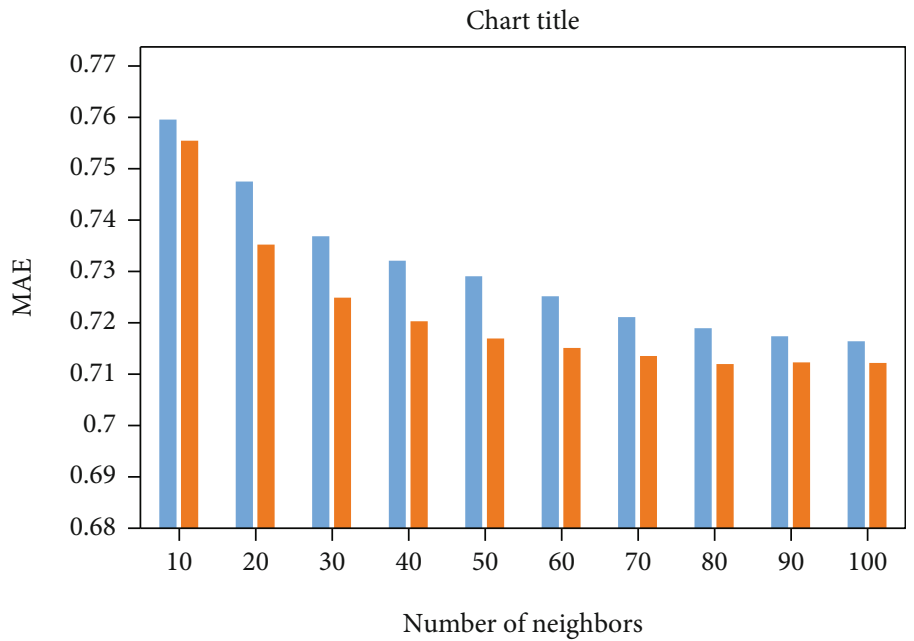

Before introducing the trust mechanism

After introducing the trust mechanism

Figure 3: Comparison of MAE before and after introducing trust mechanism.

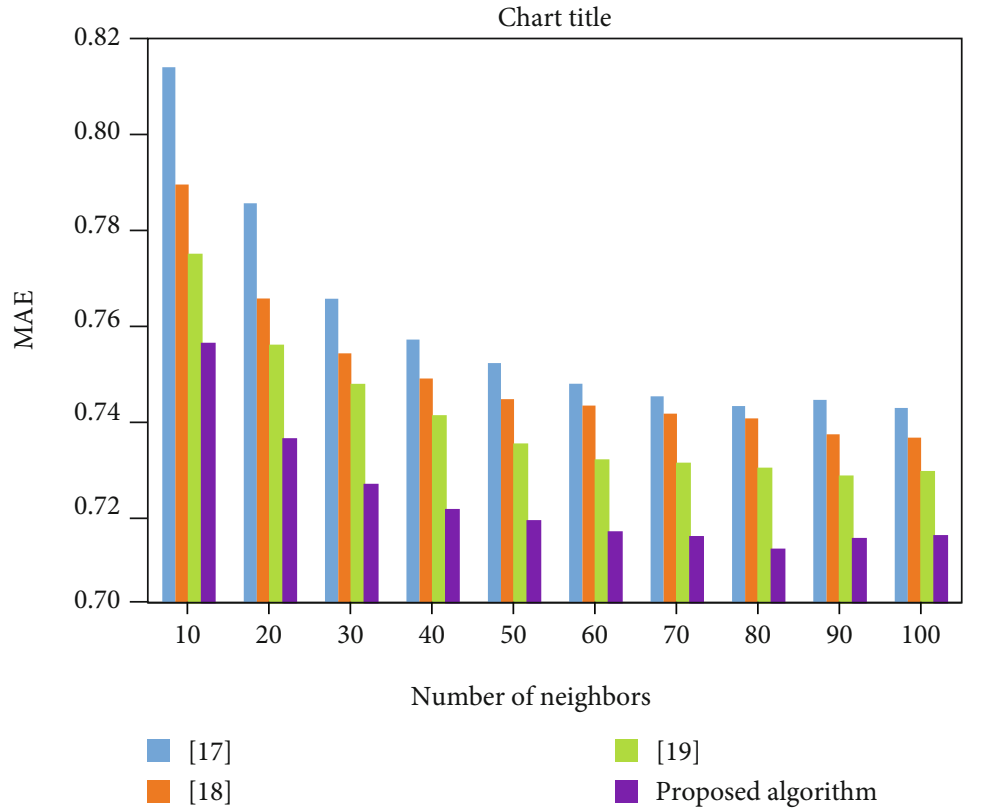

Figure 4: Comparison of MAE values of different algorithms.

statistics for $q$-type items. $N$ represents the number of all scores. $\varepsilon$ represents the weight of interest.

At the same time, using the user's interest vector combined with the cosine similarity calculation formula, the user interest similarity can be calculated for any two users $m$ and $n$.

$$
\operatorname{sim}_{b}(m, n)=\frac{\sum_{q \in Q} \text { interest }_{m, q} \times \text { interest }_{n, q}}{\sqrt{\sum_{q \in Q} \text { interest }_{m, q}^{2}} \times \sqrt{\sum_{q \in Q} \text { interest }_{n, q}^{2}}},
$$

where $Q$ represents the set of all item types.
3.2.3. Users' Mixed Similarity. In summary, the similarity obtained by the two is merged to obtain the user mixed similarity. The calculation of the two similarities is affected by the sparsity of the data in different degrees: when the number of public ratings is small, the measurement of the rating scale is susceptible to the impact of the peak of the rating, resulting in large errors in the calculation results. The interest similarity obtained based on the item type is less affected by the peak score, so the weight of the latter should be increased at this time. When the number of public scoring items is large, the accuracy of the recommendation results calculated based on user scoring is higher, so the proportion of scoring similarity should be increased at this time. 
Therefore, the threshold $d 1$ of the number of public scores is set. According to the $d 1$ size, the balanced distribution weight of the two in the case of different data sparsity is adjusted to ensure the accuracy and flexibility of the algorithm.

$$
\operatorname{sim}_{\text {mix }}(m, n)=\left\{\begin{array}{l}
\omega \frac{N_{u}}{d 1} \operatorname{sim}_{a}(m, n)+\left(1-\omega \frac{N_{u}}{d 1}\right) \operatorname{sim}_{b}(m, n), \\
N_{u}<d 1, \\
\omega \operatorname{sim}_{a}(m, n)+(1-\omega) \operatorname{sim}_{b}(m, n), \\
N_{u} \geq d 1,
\end{array}\right.
$$

where $\operatorname{sim}_{\text {mix }}(m, n)$ represents the user mixed similarity. $N_{u}$ represents the number of user scores. $\omega$ is the fusion factor. $\operatorname{sim}_{a}(m, n)$ represents corrected score similarity. $\operatorname{sim}_{b}($ $m, n)$ represents user interest similarity.

3.2.4. The Degree of Trust between Users. The analysis indicates that the trust relationship between users plays a vital role in daily life, involving all aspects. However, the description of trust is a relatively vague concept, whose cognition includes subjectivity, asymmetry, and dynamics. Traditional recommendation algorithms only consider from the point of view of scoring. But in real life, the recommendation accuracy obtained only by this method is difficult to guarantee. For example, some merchants falsify rating data to get more recommendations for their products. To reduce the impact of recommendation results on the accuracy of the scoring data, this paper introduces the trust mechanism between users. The higher the reliability between users, the higher the reliability of the recommendation data generated by them.

The trust degree between two users in the recommendation system is influenced by the interactive relationship between users. The more shared historical behavior users experience, the more trust they accumulate between each other. It is considered that there has been an interaction whenever the scoring behavior of the same item occurs between users. The interaction relationship between users can be obtained according to the proportion between the interaction times and their own historical scoring times.

$$
\operatorname{IT}(m, n)=,
$$

where $I_{m}$ and $I_{n}$ represent the set of scoring items of users $m$ and $n$, respectively. $\left|I_{m}\right|$ and $\left|I_{n}\right|$ represent the potential of the set.

The trust degree between users is affected by the interaction relationship and the interaction state. A successful interaction state is bound to strengthen trust between users. Similarly, the failed interaction state will also weaken the trust between users. This section takes the user satisfaction status as the standard to measure the success of interaction. If two users show satisfaction or dissatisfaction with a project at the same time, their interaction state will be considered to be successful. If two users have different satisfactions with the jointly evaluated items, their interaction state will be considered to be a failure. Satisfaction is calculated by comparing the scores of two users' common items with their respective average scores. If the score is higher than their own average score, it means satisfaction; otherwise, it means dissatisfaction.

The interaction states between users are as follows.

$$
S(m, n)=\left\{\begin{array}{l}
\text { sus, }\left(r_{m \mathrm{com}}-\overline{r_{m}}\right)\left(r_{n \mathrm{com}}-\overline{r_{n}}\right) \geq 0, \\
\text { fal, }\left(r_{m \mathrm{com}}-\overline{r_{m}}\right)\left(r_{n \mathrm{com}}-\overline{r_{n}}\right)<0,
\end{array}\right.
$$

where sus represents the statistical times of successful interaction. sus +1 is recorded for each successful interaction. fal indicates the statistical number of failed interactions. We record fal +1 for each interaction failure. The interaction relationship between users is calculated in combination with formula (11) to obtain the user.

$$
\operatorname{ITP}(m, n)=\operatorname{IT}(m, n) \times \frac{\text { sus }}{\text { sus }+ \text { fal }} .
$$

In the above formula, the success or failure of the interaction is considered to have the same degree of influence on trust, yet the actual situation is different. In the case of interaction failure, the difference between the two users' scores is much larger than that in the case of interaction success, which indicates that there is a great difference between the two users' satisfaction with the common score item. Therefore, it can be argued that the negative effects of failed interactions are greater. In the revision of trust degree, the score difference is added as the penalty weight affecting the interaction balance to strengthen the negative impact of failed interaction. The improved trust between users is as follows.

$$
\begin{gathered}
\operatorname{IDP}(m, n)=\operatorname{IT}(m, n) \times \sum_{e \in I_{\text {sus }}} \operatorname{sus}\left|r_{m e}-r_{n e}\right| \\
\sum_{e \in I_{\text {sus }}} \operatorname{sus}\left|r_{m e}-r_{n e}\right|+\sum_{e \in I_{\text {fal }}} \mathrm{fal}\left|r_{m e}-r_{n e}\right|
\end{gathered}
$$

where $\operatorname{IDP}(m, n)$ represents the trust degree of user $m$ to user $n . I_{\text {sus }}$ represents the set of successful interactions. $I_{\text {fal }}$ represents the set of interaction failures. $r_{m e}$ and $r_{n e}$ represent the scores of user $m$ and user $n$ on item $e$, respectively. Formula (9) satisfies the asymmetric principle of trust, that is, $\operatorname{IDP}(m, n) \neq \operatorname{IDP}(n, m)$.

If the hybrid similarity between target user $m$ and target user $n$ is higher, and $m$ feels that $n$ is trustworthy, then $m$ is more likely to adopt $n$ 's suggestion. Therefore, when establishing the neighbor user set $U_{s}$, the hybrid similarity and trust degree are arranged in reverse order according to the value, and the top $k$ users with the highest comprehensive value are selected to establish the neighbor user set. The prediction score $P_{m, e}$ is generated.

$$
P_{m, e}=\overline{r_{m}}+\frac{\sum_{n \in U_{s}} \theta \operatorname{sim}_{\text {mix }}(m, n)+(1-\theta) \operatorname{IDP}(m, n)\left(r_{n, e}-\overline{r_{n}}\right)}{\sum_{n \in U_{s}}\left|\operatorname{sim}_{\text {mix }}(m, n)+(1-\theta) \operatorname{IDP}(m, n)\right|} .
$$


In the formula, the value of $\theta$ is determined by the adaptive model, and the value range of $\theta$ is $[0,1]$.

$$
\theta=\frac{\operatorname{sim}_{\text {mix }}^{2}(m, n)}{\operatorname{sim}_{\text {mix }}^{2}(m, n)+\operatorname{IDP}(m, n)^{2}(m, n)} .
$$

3.3. Improved Algorithm Based on the Item and Its Characteristics. Section 3.2 generates neighbor data according to multidimensional user scores and user characteristic data. However, the similarity of project features is not taken into account; besides, the recommendation accuracy is not very good. In this section, multidimensional user similarity and trust are used to generate user neighbor data sets as training sets, and item scores and features are taken into account to improve the traditional slope one algorithm. Users and items are taken into account, which make the final prediction score more accurate and the recommendation effect better. The calculation method of item feature similarity is similar to that of user feature similarity.

$$
\begin{gathered}
\operatorname{Dis}(m, n)=\sqrt{\sum_{l=1}^{L}\left(m_{l}-n_{l}\right)^{2},} \\
\operatorname{SimItem}(m, n)=\frac{1}{1+\operatorname{Dis}(m, n)} .
\end{gathered}
$$

In the formula above, $L$ represents the number of attribute features of the item. $m_{l}$ represents the $l$ th attribute of item $m . n_{l}$ represents the $l$ th attribute value of item $n$. The similarity of item features is calculated as the method of calculating the similarity of similar user features.

Item similarity is also calculated by the Pearson formula.

$$
\operatorname{SimItem}(m, n)=\frac{\sum_{v=1}^{V}\left(R_{v, m}-A_{m}\right) \times\left(R_{v, n}-A_{n}\right)}{\sqrt{\sum_{v=1}^{V}\left(R_{v, m}-A_{m}\right)^{2} \times \sum_{v=1}^{V}\left(R_{v, n}-A_{n}\right)^{2}}} .
$$

In the formula, $A_{m}$ refers to the average score of all users' nearest neighbors of user $v$ on item $m . V$ is the number of recent neighbors for all items of project $m . R_{v, n}$ refers to the score of user $v$ on item $n$. $\operatorname{SimItem}(m, n)$ refers to the score similarity of item $m$ and item $n$.

Based on the recommendation set of the user's nearest neighbor set, the item's nearest neighbor set is calculated, and the recommendation is finally generated.

$p(v)_{n}=\frac{\sum_{m=1}^{V}\left(1 / \operatorname{card}\left(R_{n}\right)\right) \sum_{m \in R_{n}}\left(\operatorname{dev}_{m, n}+v_{m}\right) \times \operatorname{SimItem}(m, n)}{\sum_{m=1}^{V} \operatorname{SimItem}(m, n)}$,

where card $\left(R_{n}\right)$ represents the number of elements in $R_{n}$ . $\operatorname{dev}_{m, n}$ represents the average deviation between items $m$ and $n . v_{m}$ represents user $v$ 's rating of item $m$.

3.4. Recommended Steps for Interior Design Schemes to Improve Collaborative Filtering Algorithm. Step 1: collect the relevant historical data of indoor project design and process it to remove some wrong data.

Step 2: extract the user features and project features of interior project design, which form the corresponding feature set. Then, mark all users and scoring data in the matrix.

Step 3: by introducing the fuzzy logic method, the user's fuzzy preference is obtained by enriching the score difference between users. Then, the score similarity is modified based on fuzzy preference differences. The user modified score similarity $\operatorname{sim}_{a}(m, n)$ is obtained by combining the Jaccard similarity coefficient.

Step 4: calculate the proportion between the mean scores of various types of items and the sum of the mean scores of all items, as well as the ratio between the scoring times of various items and the total scoring times of items. The sum of the two is calculated to obtain the user's interest vector. Combined with cosine similarity, $\operatorname{sim}_{b}(i, j)$ of user interest similarity is calculated.

Step 5: calculate the similarity between Step 3 and Step 4 based on different factors. The weight fusion is carried out by combining formula (8) to obtain the mixed similarity $\operatorname{sim}_{\text {mix }}(m, n)$. Considering that only using the predicted score generated by $\operatorname{sim}_{\text {mix }}(m, n)$ for project recommendation may lead to the problem of single recommendation factor and poor recommendation accuracy, the trust relationship between users is introduced on this basis. The first $k$ users with the highest comprehensive value are obtained to establish the neighbor user set.

Step 6: use multidimensional user similarity and trust to generate user neighbor data set as the training set. Formula (17) is used to score items.

Step 7: arrange the predicted scores in descending order and recommend the top $N$ interior items with the highest predicted scores to the target users for the projects not scored by the target users.

\section{Experimental Results}

4.1. Experimental Data and Experimental Environment. Some interior design item data sets are used as test objects to test the performance of collaborative filtering technology in the interior design project recommendation method proposed in this paper.

In the researches on the collaborative filtering algorithm, most of them use the MovieLens website data set maintained by the GroupLens laboratory. This data set contains more comprehensive user rating information of movies covering all time points. And it is still being maintained at present. In this paper, the historical behavior data of users are processed into the same format with reference to the MovieLens data set. The data set of user rating on the design scheme, which is recorded by the icolor server of the company's project background home decoration website for one month, is selected as the experimental data. The data set contains $12,829,401$ scoring information of 16,427 home decoration design schemes by 13,512 website users, ranging from 1 to 5 points.

The experimental environment settings are as follows: (1) hardware environment: Intel 7 core CPU @ $2.69 \mathrm{GHz}$, 
12 GB memory; (2) software environment: Win10 operating system with Java programming language.

4.2. Experimental Indicators. In most studies, the commonly used indicators are as follows, which are applied to measure the effect of the collaborative filtering algorithm conveniently.

The mean absolute error (MAE) is the average of the absolute values of errors. It uses the deviation between the item score predicted by the recommendation algorithm and the user's actual score value to measure the accuracy of the recommendation. The range of this value is $[0,1]$. The smaller the MAE value, the higher the accuracy of the recommendation algorithm and the better the recommendation quality.

$$
\mathrm{MAE}=\frac{1}{\operatorname{num}(R)} \sum_{m \in R}\left(p_{v m}-r_{v m}\right) .
$$

\subsection{Analysis of Experimental Results}

4.3.1. Determination of Interest Vector Weight $\varepsilon$. Interest vector weight $\alpha$ is mainly used to adjust the ratio between different types of items and the total score value and adjust the ratio between the number of different types of items and the total score items. By adjusting the proportion of the two, different interest vectors interest ${ }_{m, n}$ can be obtained. Then, interest similarity between users is calculated according to interest vector $\operatorname{sim}_{b}(m, n)$. During the experiment, $\varepsilon$ increases with a step size of 0.1 , and the value range is $[0,1]$. The neighbor set $\mathrm{Nei}_{\text {col }}$ takes 10 steps and ranges from 10 to 60. Multiple groups of predicted scores are generated according to the calculation, and the MAE of the corresponding real score and predicted score is obtained. The statistical results are shown in Table 1.

It can be seen from Table 1 that MAE decreases with the increase of the number of neighbor sets, indicating that the recommendation accuracy is significantly affected by data sparsity. As can be seen from Table 2, when $k=60$ and $\varepsilon=$ 0.7 , the average absolute error between the predicted score and the real score is minimum and reaches the optimal value. Therefore, the unknown parameter $\varepsilon$ is 0.7 .

4.3.2. Determination of Fusion Factor $\omega$. The function of dynamic fusion factor $\omega$ is to dynamically adjust the proportion of two similarity algorithms in mixed similarity according to the difference of data sparsity. In the experiment, the number of neighbor sets $k$ and fusion factor $\omega$ is uniformly changed by step size, and the change curves of multiple sets of prediction scores and MAE are calculated. The experimental results are shown in Table 2.

It can be seen from Table 2 that as the number of neighbor sets increases, the MAE difference decreases gradually. When the set number $k$ is 60 and the dynamic factor $\omega$ is 0.9 , MAE obtains the optimal value, so $\omega=0.9$ in this paper.

4.3.3. Comparison of Algorithm Performance before and after Introducing Trust Mechanism. The smaller the MAE value obtained by the two algorithms before and after the intro- duction of trust mechanism, the higher the accuracy of the algorithm. According to the optimal values $\varepsilon=0.7$ and $\omega=$ 0.9 obtained in experiment 1 , the neighbor user sets before and after the introduction of trust mechanism are obtained. The neighbor set number $k$ is incremented with the step size of 10, and two MAE groups corresponding to the two algorithms are calculated. The experimental results are shown in Figure 3.

It can be seen from the analysis in Figure 3 that with the increase of the number of neighbor users, the MAE change trend obtained by the algorithm after the introduction of trust mechanism shows a downward trend from sharp to slow compared with the algorithm before the introduction. It can be seen that this algorithm has higher recommendation accuracy when the data sparsity is high. With the increase of the number $k$ of neighbor users, the accuracy of the algorithm with trust mechanism is improved to a certain extent compared with the algorithm without the trust mechanism. This verifies that the trust mechanism is introduced to optimize the performance of the algorithm.

4.3.4. Comparison of Accuracy between the Proposed Algorithm and Other Algorithms. The accuracy comparison of several algorithms also adopts the method of comparative experiment. The comparative algorithms include the collaborative filtering recommendation algorithm with cosine similarity correction score in literature [17], the collaborative filtering algorithm based on improved time function and user similarity proposed in literature [18], and the collaborative filtering recommendation algorithm based on heuristic similarity measurement in literature [19]. MAE corresponding to each algorithm was calculated. The experimental results are shown in Figure 4.

The MAE of the proposed algorithm is smaller than that of the other three algorithms. The experimental results are analyzed as follows.

By introducing user interest, trust mechanism and item score, the algorithm in this paper has obvious advantages in the case of data sparsity compared with other algorithms. When $k=10$, MAE of this algorithm is 0.7551 . Compared with literature [17-19], MAE of the proposed algorithm is reduced by $7.23 \%, 4.30 \%$, and $2.45 \%$, respectively. When the number of neighbor users increase, the recommendation accuracy of each algorithm is improved to varying degrees. When the number of neighbor sets $k=80$, MAE of this algorithm gets the minimum value, reaching 0.7113. Compared with literature [17-19], MAE of the proposed algorithm is reduced by $4.12 \%, 3.78 \%$, and $2.37 \%$, respectively. In conclusion, compared with other collaborative filtering algorithms, the recommendation accuracy of the proposed algorithm is significantly improved, and the performance improvement of recommendation accuracy is more obvious in the case of data sparsity.

\section{Conclusion}

Interior design, the quality of which is critical, is the current hot spot. Therefore, the recommendation method of the interior design item has become a highly concerned issue. 
Due to the shortcomings of current interior design recommendation methods such as large errors and long time consumption, an interior design project recommendation method based on an improved collaborative filtering algorithm was designed to obtain ideal interior design project recommendation results. The following conclusions can be drawn from the experimental data. (1) Due to the fusion of multilayer hybrid similarity, trust mechanism, and extraction of interior design item features, the algorithm in this paper can achieve an accurate recommendation of interior design projects, and its performance is better than that of other algorithms. This algorithm controls the error rate of interior design project recommendation within the scope of practical application, solving the problem of high error rate in current interior design project recommendation methods. (2) The interior design item recommendation method based on improved collaborative filtering technology can be applied to solving problems with similar characteristics in other fields, which has very wide application value. In the future research work, it is necessary to focus on the problem of starting the algorithm. Moreover, we will incorporate some intelligent algorithms such as deep learning, federated learning, and mobile edge computing techniques into the considered system [20-27], in order to further enhance the system performance.

\section{Data Availability}

The labeled data set used to support the findings of this study is available from the corresponding author upon request.

\section{Conflicts of Interest}

The author declares no competing interests.

\section{Acknowledgments}

This study was supported by the Scientific Research Program of Shaanxi Provincial Department of Education: Research on the Innovative Application of Chinese Traditional Decorative Elements in Urban Public Space-Taking Xi'an as an Example (No. 19jk0896).

\section{References}

[1] Y. Yu, X. Wang, R. Y. Zhong, and G. Q. Huang, "E-commerce logistics in supply chain management," Industrial Management \& Data Systems, vol. 117, no. 10, pp. 2263-2286, 2017.

[2] L. Haibing and K. Yuyue, "Business model innovation of Qingdao Youzhu Decoration Company," EDP Sciences, vol. 214, article 02046, 2020.

[3] Y. S. Chang, K. J. Hu, C. W. Chiang, and A. Lugmayr, "Applying mobile augmented reality (AR) to teach interior design students in layout plans: evaluation of learning effectiveness based on the ARCS model of learning motivation theory," Sensors, vol. 20, no. 1, pp. 105-130, 2020.

[4] S. Zhong, P. Tan, T. Fu, and Y. Ji, "Product design model for Ecommerce Cantonese porcelain based on user perceptual image in China," in Human-Computer Interaction. Human
Values and Quality of Life. HCII 2020, Lecture Notes in Computer Science, M. Kurosu, Ed., pp. 350-364, Springer, Cham, 2020.

[5] J. Zhang, X. Hu, Z. Ning et al., "Energy-latency tradeoff for energy-aware offloading in mobile edge computing networks," IEEE Internet of Things Journal, vol. 5, no. 4, pp. 2633-2645, 2018.

[6] S. R. Pardhi and K. H. Wanjale, "Extraction and retrieval of furniture from designing decoration and furniture database," in 2017 International Conference on Computer Communication and Informatics (ICCCI), pp. 1-6, IEEE, Coimbatore, India, 2017.

[7] X. Xu, "Home decoration comprehensive application web design and analysis," International Journal of Science, vol. 6, no. 4, pp. 36-41, 2019.

[8] Z. Cui, X. Xu, F. Xue et al., "Personalized recommendation system based on collaborative filtering for IoT scenarios," IEEE Transactions on Services Computing, vol. 13, no. 4, pp. 685695, 2020.

[9] X. Chen, S. Li, H. Li, S. Jiang, Y. Qi, and L. Song, "Generative adversarial user model for reinforcement learning based recommendation system," in International Conference on Machine Learning, pp. 1052-1061, Long Beach, California, USA, 2019.

[10] L. Jiang, Y. Cheng, L. Yang, J. Li, H. Yan, and X. Wang, "A trust-based collaborative filtering algorithm for E-commerce recommendation system," Journal of Ambient Intelligence and Humanized Computing, vol. 10, no. 8, pp. 3023-3034, 2019.

[11] B. Hong and M. Yu, "A collaborative filtering algorithm based on correlation coefficient," Neural Computing and Applications, vol. 31, no. 12, pp. 8317-8326, 2019.

[12] M. Ayub, M. A. Ghazanfar, T. Khan, and A. Saleem, "An effective model for Jaccard coefficient to increase the performance of collaborative filtering," Arabian Journal for Science and Engineering, vol. 45, no. 12, pp. 9997-10017, 2020.

[13] S. Jiang, S. C. Fang, Q. An, and J. E. Lavery, "A sub-one quasinorm-based similarity measure for collaborative filtering in recommender systems," Information Sciences, vol. 487, pp. 142-155, 2019.

[14] Z. Ning, X. Hu, Z. Chen et al., “A cooperative quality-aware service access system for social Internet of vehicles," IEEE Internet of Things Journal, vol. 5, no. 4, pp. 2506-2517, 2017.

[15] B. Alhijawi and Y. Kilani, "A collaborative filtering recommender system using genetic algorithm," Information Processing \& Management, vol. 57, no. 6, article 102310, 2020.

[16] Z. Li, "Interior design recommendation technology based on collaborative filtering algorithm," Modern Electronics Technique., vol. 43, no. 13, pp. 176-179, 2020.

[17] R. Zhang and X. Qian, "A collaborative filtering recommendation algorithm with revised rating by cosine similarity," Computer Engineering and Science., vol. 42, no. 6, pp. 1096-1105, 2020.

[18] W. Zhang, X. Zhou, and W. Yuan, "Collaborative filtering algorithm based on improved time function and user similarity," Journal of physics: conference series, vol. 1757, no. 1, article 012080, 2021.

[19] R. Shaw, D. K. Agrawal, and B. K. Patra, "An effective similarity measure for improving performance of user based collaborative filtering," in IEEE EUROCON 2021-19th International Conference on Smart Technologies, pp. 209-215, Lviv, Ukraine, 2021. 
[20] J. Xia and D. Deng, “A note on implementation methodologies of deep learning-based signal detection for conventional MIMO transmitters," IEEE Transactions on Broadcasting, vol. 66, no. 3, pp. 744-745, 2020.

[21] L. He and K. He, "Efficient Memory-Bounded Optimal Detection for GSM-MIMO Systems," IEEE Transactions on Communications, vol. 69, no. 1, pp. 101-112, 2021.

[22] H. Xiping, J. Cheng, M. Zhou et al., "Emotion-aware cognitive system in multi-channel cognitive radio ad hoc networks," IEEE Communications Magazine, vol. 56, no. 4, pp. 180-187, 2018.

[23] W. Zhou, "PSO based offloading strategy for cache-enabled mobile edge computing UAV networks," Cluster Computing, vol. 2021, no. 24, pp. 1-13, 2021.

[24] L. Chen, "Intelligent ubiquitous computing for future UAVenabled MEC network systems," Cluster Computing, vol. 2021, no. 25, pp. 1-10, 2021.

[25] L. He and K. He, “Towards optimally efficient search with deep learning for large-scale MIMO systems," IEEE Transactions on Communications, no. 99, pp. 1-12, 2022.

[26] S. Tang and L. Chen, "Computational intelligence and deep learning for next-generation edge-enabled industrial IoT," IEEE Transactions on Network Science and Engineering, no. 99, pp. 1-12, 2022.

[27] L. Chen, "Physical-layer security on mobile edge computing for emerging cyber physical systems," Computer Communications, no. 99, pp. 1-10, 2022. 